\section{(6) OPEN ACCESS}

\title{
Pro-inflammatory effects of e-cigarette vapour condensate on human alveolar macrophages
}

\author{
Aaron Scott, ${ }^{1}$ Sebastian T Lugg, ${ }^{1}$ Kerrie Aldridge, ${ }_{1}^{1}$ Keir E Lewis, ${ }^{2}$ Allen Bowden, ${ }^{3}$ \\ Rahul Y Mahida, ${ }^{1}$ Frances Susanna Grudzinska, ${ }^{1}$ Davinder Dosanjh, ${ }^{1}$ Dhruv Parekh, ${ }^{1}$ \\ Robert Foronjy, ${ }^{4}$ Elizabeth Sapey, ${ }^{1}$ Babu Naidu, ${ }^{1}$ David R Thickett ${ }^{1}$
}

\begin{abstract}
- Additional material is published online only. To view please visit the journal online (http://dx.doi.org/10.1136/ thoraxjn-2018-211663).
\end{abstract}

${ }^{1}$ Birmingham Acute Care Research Group Institute of Inflammation and Ageing (IIA), University of Birmingham, Birmingham, UK ${ }^{2}$ College of Medicine, Swansea University, Swansea, UK ${ }^{3}$ Analytical Facility, School of Chemistry, University of Birmingham, Birmingham, UK ${ }^{4}$ Division of Pulmonary and Critical Care Medicine, Department of Medicine, SUNY Downstate Medical Center, Brooklyn, New York, USA

\section{Correspondence to} Dr David R Thickett, Institute of Inflammation and Ageing, University of Birmingham, d.thickett@bham.ac.uk

Received 8 February 2018 Revised 16 May 2018 Accepted 11 June 2018 Published Online First 13 August 2018

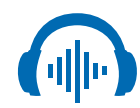

Listen to Podcast www.thorax.bmj.com Birmingham B15 2TH, UK;

ABSTRACT
Objective Vaping may increase the cytotoxic effects of e-cigarette liquid (ECL). We compared the effect of unvaped ECL to e-cigarette vapour condensate (ECVC) on alveolar macrophage (AM) function.

Methods AMs were treated with ECVC and nicotinefree ECVC (nfECVC). AM viability, apoptosis, necrosis, cytokine, chemokine and protease release, reactive oxygen species (ROS) release and bacterial phagocytosis were assessed.

Results Macrophage culture with ECL or ECVC resulted in a dose-dependent reduction in cell viability. ECVC was cytotoxic at lower concentrations than ECL and resulted in increased apoptosis and necrosis. nfECVC resulted in less cytotoxicity and apoptosis. Exposure of AMs to a sub-lethal $0.5 \%$ ECVC/nfECVC increased ROS production approximately 50 -fold and significantly inhibited phagocytosis. Pan and class one isoform phosphoinositide 3 kinase inhibitors partially inhibited the effects of ECVC/nfECVC on macrophage viability and apoptosis. Secretion of interleukin 6 , tumour necrosis factor $\alpha, C X C L-8$, monocyte chemoattractant protein 1 and matrix metalloproteinase 9 was significantly increased following ECVC challenge. Treatment with the anti-oxidant $\mathrm{N}$-acetyl-cysteine (NAC) ameliorated the cytotoxic effects of ECVC/nfECVC to levels not significantly different from baseline and restored phagocytic function.

Conclusions ECVC is significantly more toxic to AMs than non-vaped ECL. Excessive production of ROS, inflammatory cytokines and chemokines induced by e-cigarette vapour may induce an inflammatory state in AMs within the lung that is partly dependent on nicotine. Inhibition of phagocytosis also suggests users may suffer from impaired bacterial clearance. While further research is needed to fully understand the effects of e-cigarette exposure in humans in vivo, we caution against the widely held opinion that e-cigarettes are safe.

\section{INTRODUCTION}

Check for updates

(c) Author(s) (or their employer(s)) 2018. Re-use permitted under CC BY-NC. No commercial re-use. See rights and permissions. Published by BMJ.

To cite: Scott A, Lugg ST, Aldridge $\mathrm{K}$, et al. Thorax 2018;73:1161-1169.
Electronic cigarettes, also known as electronic nicotine delivery systems (ENDS), were introduced over a decade ago and since 2010 the inhalation of e-cigarette vapour or 'vaping' has risen exponentially in both smokers and ex-smokers. ${ }^{1}$ There is a significant body of published material on ENDS/e-cigarettes and despite varying opinions their main effects remain controversial. They may be a useful tool for reducing traditional cigarette smoking but for many they are simply a replacement nicotine

\section{Key messages}

What is the key question?

- Do e-cigarettes have a negative impact on alveolar macrophage viability and function?

What is the bottom line?

- Vapourised e-cigarette fluid is cytotoxic, proinflammatory and inhibits phagocytosis in alveolar macrophages.

Why read on?

- This work demonstrates a nicotine dependent and independent effect and also examines how these effects may be abrogated.

delivery method. As such they may precipitate a new public health problem. ${ }^{2}$ The public perception is that they are less of a health hazard than conventional cigarette smoking, yet the long-term effects of e-cigarettes remain to be elucidated. ${ }^{2}$

E-cigarettes have developed significantly in the last decade, increasing in complexity and capacity. They are now considered to be in the fourth generation, comprising highly modifiable devices capable of modulating the energy input used to generate vapour. Using ever increasing energy input, sub-ohm atomiser resistances and custom mixtures for electronic cigarette liquid (ECL), the effect of user exposure is becoming more uncertain and potentially a new health hazard. ${ }^{34}$

Prior to vaping, ECL is composed of humectants such as vegetable glycerin (VG) and propylene glycol (PG) with or without nicotine. Several potentially cytotoxic metal and silicate particles are present in e-cigarette vapour equal to or exceeding the levels found in traditional cigarette smoke. ${ }^{56}$ Much of the current literature has focused on the effect of non-vapourised ECL or ECL condensate. However, such studies do not fully reflect the potential effect on an e-cigarette user as, importantly, the process of vaping itself causes changes in the chemical composition of ECL. ${ }^{7-11}$ Recently, some studies have attempted a more physiological approach using aqueous extract systems similar to those used to create traditional cigarette smoke extract (CSE), ${ }^{12}$ although this also results in considerable dilution. In vivo studies have also been carried out using whole animal aerosol exposure systems, without vaping, which have also predominantly focused 
on the effects on the lungs-. ${ }^{13} 14$ These studies showed exposure to unvaped fluid increased secretion of inflammatory markers, induced airway hyper-reactivity and caused lung tissue degradation in chronic exposure. ${ }^{13} 14$ These studies demonstrated the potential negative impact of e-cigarette fluid exposure, however the proven change in composition caused by the vaping process has not been factored in these models.

For this study, we developed a novel system to generate e-cigarette vapour condensate (ECVC) to be a more physiological method of exposure. We hypothesised the change in chemical composition caused by vaping would increase cytotoxicity and moreover the presence of nicotine would exacerbate any cytotoxic and pro-inflammatory effects. Alveolar macrophages (AMs) are a unique lung cell population that eliminate airborne irritants and infectious agents, while also coordinating the initiation of resolution of lung inflammation. ${ }^{15}$

Disturbances in AM function could therefore increase the risk of infection and enhance susceptibility to chronic obstructive pulmonary disease (COPD). We also assessed the effects of our ECVC with and without nicotine on human AMs to determine if this is a key component and determine whether anti-oxidants abrogate any of the effects.

\section{METHODS}

Detailed methods are provided in the online supplement.

\section{ECVC preparation}

ECVC was prepared using a novel method employing six tracheal suction traps (Unomedical, Denmark) connected in series and cooled in a dry ice/methanol bath (see online supplementary figure E1a). We calculated the optimal puff duration of $3 \mathrm{~s}$ every $30 \mathrm{~s}$ based on published data. ${ }^{16-21}$ This allows time for the vapour to condense between each puff and prevented overheating of the device; $1.4 \mathrm{~mL}$ of ECL/nicotine-free ECL (nfECL) was vaped from each device. On completion, suction traps were normalised to room temperature and spun at $1500 \mathrm{~g}$ for $10 \mathrm{~min}$ to collect the condensate.

\section{E-cigarette devices}

We chose a second-generation END, popular in the UK, to produce condensate (Kanger Ltd, Shenzhen, China; see online supplementary figure E1b). The devices were fitted with a standard $650 \mathrm{mAh}$ battery with a fresh $1.8 \mathrm{Ohm}$ coil head (atomiser) for each preparation.

\section{E-cigarette liquids}

ECLs with and without pharmaceutical grade nicotine were obtained from American E-liquids Store (Milwaukee County Research Park, Wauwatosa, WI, USA), which adheres to US Food and Drug Administration (FDA) approved good manufacturing standards and has been used in previous animal exposure studies. ${ }^{13}$ To avoid confounders, only flavourless liquids were used. Nicotine containing ECL was $36 \mathrm{mg} / \mathrm{mL}$, nfECL was simply a 50:50 mixture of PG:VG.

\section{Alveolar and THP-1 macrophages}

AMs from eight never smokers, five men and three women, with normal spirometry and no history of asthma/COPD were obtained by repeated saline lavage from non-affected lung resection specimens (see online supplement for full extraction methods).

THP-1 human monocytic leukaemia cells (European Collection of Cell Cultures) were differentiated into macrophages by stimulation with $0.2 \mathrm{mM}$ phorbol 12 -myristate 13 -acetate (PMA) for 24 hours. Adhered cells were rested in RPMI for 3 days before use. ${ }^{22}$

Gas chromatography-flame ionisation detector assessment of nicotine content

Gas chromatography-flame ionisation detector (GC-FID) assessment was performed by the University of Birmingham Chemistry Department to determine nicotine concentration. L-Nicotine standard (\#10337220 Fisher Scientific, UK) was used as a reference standard for quantification.

\section{Cellular methods}

Viability was assessed using CellTiter 96 AQueous One Solution (Roche, UK). Apoptosis was assessed by flow cytometry using an Annexin V assay (BD Biosciences, UK) in combination with the vital dye propidium iodide (PI) (Sigma-Aldrich, UK). CXCL-8, interleukin (IL)-6, monocyte chemoattractant protein (MCP)-1, tumour necrosis factor (TNF)- $\alpha$ and matrix metalloproteinase (MMP)-9 levels in cell-free supernatants were quantified using commercially available ELISA kits (Biotechne, UK). Reactive oxygen species (ROS) were measured using DCFDA assay (Abcam ab113851) according to the manufacturer's instructions. Phagocytosis assay was carried out using pHrodo Red Escherichia coli or Staphylococcus aureus BioParticles (Invitrogen, UK) according to the manufacturer's instructions.

\section{Statistical analyses}

Statistical analyses were performed using GraphPad PRISM 6.0 software package (San Diego, California, USA). Results are expressed as the median with IQR, unless specified otherwise. All results are representative of at least eight independent experiments performed in duplicate. Differences between multiple treatments were compared by the Kruskal-Wallis test followed by Dunn's multiple comparison post-test correction. A P-value $\leq 0.05$ was considered to represent a statistically significant difference.

\section{RESULTS}

\section{Inter-batch variability of unvaped ECL}

All condensates used in this study were generated from two batches of ECL. GC-FID data (table 1A) suggested actual nicotine content of ECL was $31.0 \mathrm{mg} / \mathrm{mL}$ and $30.7 \mathrm{mg} / \mathrm{mL}$ for batch 1 and 2 respectively. No nicotine was detected in nicotine-free liquids.

Validation of our model system of condensing vaped ECL

Detailed validation of our model of condensing vaped ECL is available in the online supplement (online supplementary figure E2). The model system proved both reliable and reproducible in terms of volume of recovery $(60.8 \%)$, nicotine content $(87 \%)$, as well as particulates (OD at $370 \mathrm{~nm}$, table $1 \mathrm{~B})$. There was no significant variance in biological activity between each fresh preparation (online supplementary figure E2), however biological activity was lost over time with storage at $-80^{\circ} \mathrm{C}$ (online supplementary figure $\mathrm{E} 3, \mathrm{P}<0.0001$ ).

\section{ECVC is significantly more cytotoxic to AM than ECL}

AMs were exposed to ECL and ECVC for 24hours, and produced a dos- dependent reduction in viability (figure 1). Unvaped ECL/nfECL effect on viability varied significantly compared with untreated control (UTC) following 2.5\% (v/v) challenge (figure 1A, B): ECL: 78.8\% viable (IQR 72.3\%-87.6\%, $\mathrm{P}<0.001$ ), nfECL: $84.6 \%$ viable (IQR 83.9\%-87.9\%, $\mathrm{P}<0.001$ ). 
Table 1 Inter-batch variance. (A) Inter-batch variation between ECLs obtained from American e-liquids store was assessed by GC-FID to determine nicotine content. (B) Inter-batch variation between preparations of condensate was assessed by measurement of physical characteristics, including volume recovered, nicotine recovery, optical density and cytotoxic potential following a 24 hour challenge with each condensate. Preparations $1-3$ were produced from ECL batch 1, preparations 4-6 were produced from ECL batch 2

\begin{tabular}{|c|c|c|}
\hline \multirow[b]{2}{*}{ (A) } & \multicolumn{2}{|c|}{ ECL batch } \\
\hline & 1 & 2 \\
\hline Expected nicotine $(\mathrm{mg} / \mathrm{mL})$ & 36.00 & 36.00 \\
\hline Observed nicotine (mg/mL) & 31.00 & 30.70 \\
\hline Disparity & 13.89 & 14.72 \\
\hline Mean OD $370 \mathrm{~nm}$ & 0.22 & 0.21 \\
\hline
\end{tabular}

\begin{tabular}{|c|c|c|c|c|c|c|c|c|c|}
\hline \multirow[b]{2}{*}{ (B) } & \multicolumn{6}{|c|}{ Condensate preparation } & \multirow[b]{2}{*}{ Mean } & \multirow[b]{2}{*}{ SD } & \multirow[b]{2}{*}{ CV \% } \\
\hline & 1 & 2 & 3 & 4 & 5 & 6 & & & \\
\hline Input volume $(\mu \mathrm{L})$ & 1400 & 1400 & 1400 & 1400 & 1400 & 1400 & 1400 & 0 & 0 \\
\hline Recovery (\%) & 60.7 & 62.1 & 59.3 & 61.4 & 57.9 & 63.6 & 60.8 & 2.0 & 3.4 \\
\hline Nicotine (mg/mL) & 26 & 24.8 & 28 & 23.9 & 26.8 & 29.6 & 26.5 & 2.1 & 7.9 \\
\hline
\end{tabular}

$\mathrm{ECL}$, e-cigarette liquid; GC-FID, gas chromatography-flame ionisation detector; OD, optical density.
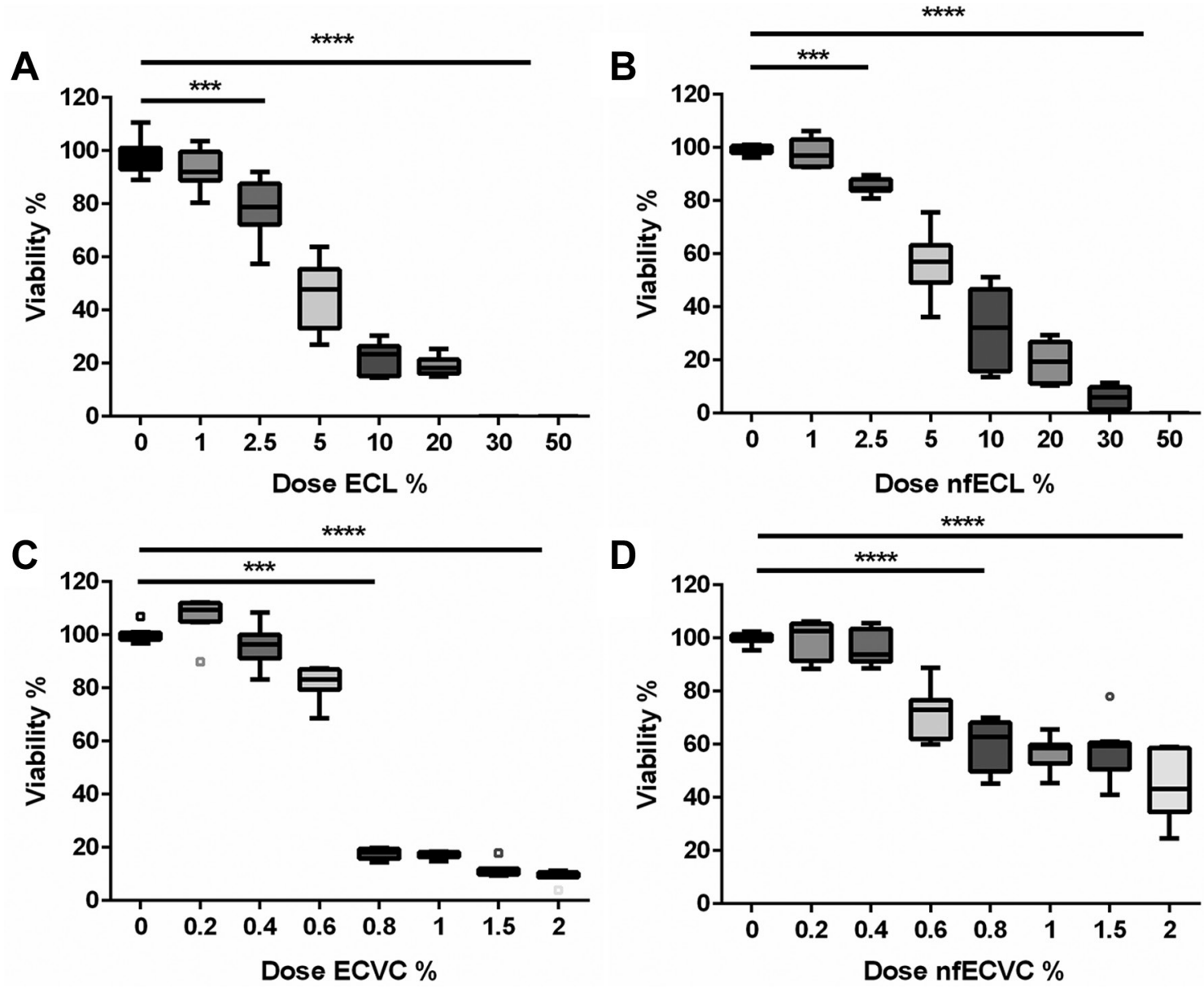

Figure 1 Effect of e-cigarette vapour condensate (ECVC) and -cigarette liquid (ECL) on alveolar macrophage viability. Viability was assessed by 4 hour incubation with cell titre aqueous assay following 24 hour exposure to a range of doses with (A) ECL, (B) nicotine-free ECL (nfECL), (C) ECVC, (D) nicotine-free ECVC (nfECVC). Graphs presented as median with IQR of eight independent experiments. The central horizontal line on each box plot represents the median, the upper and lower horizontal lines represent the first (Q1) and third (Q3) quartiles, respectively, and the vertical lines represent the range of values within the limits $\mathrm{Q} 1-1.5$ (Q3-Q1) and Q3-1.5 (Q3-Q1). $n=8,{ }^{*} P<0.05,{ }^{* *} P<0.01,{ }^{* * *} P<0.001,{ }^{* * * *} P<0.0001$. 


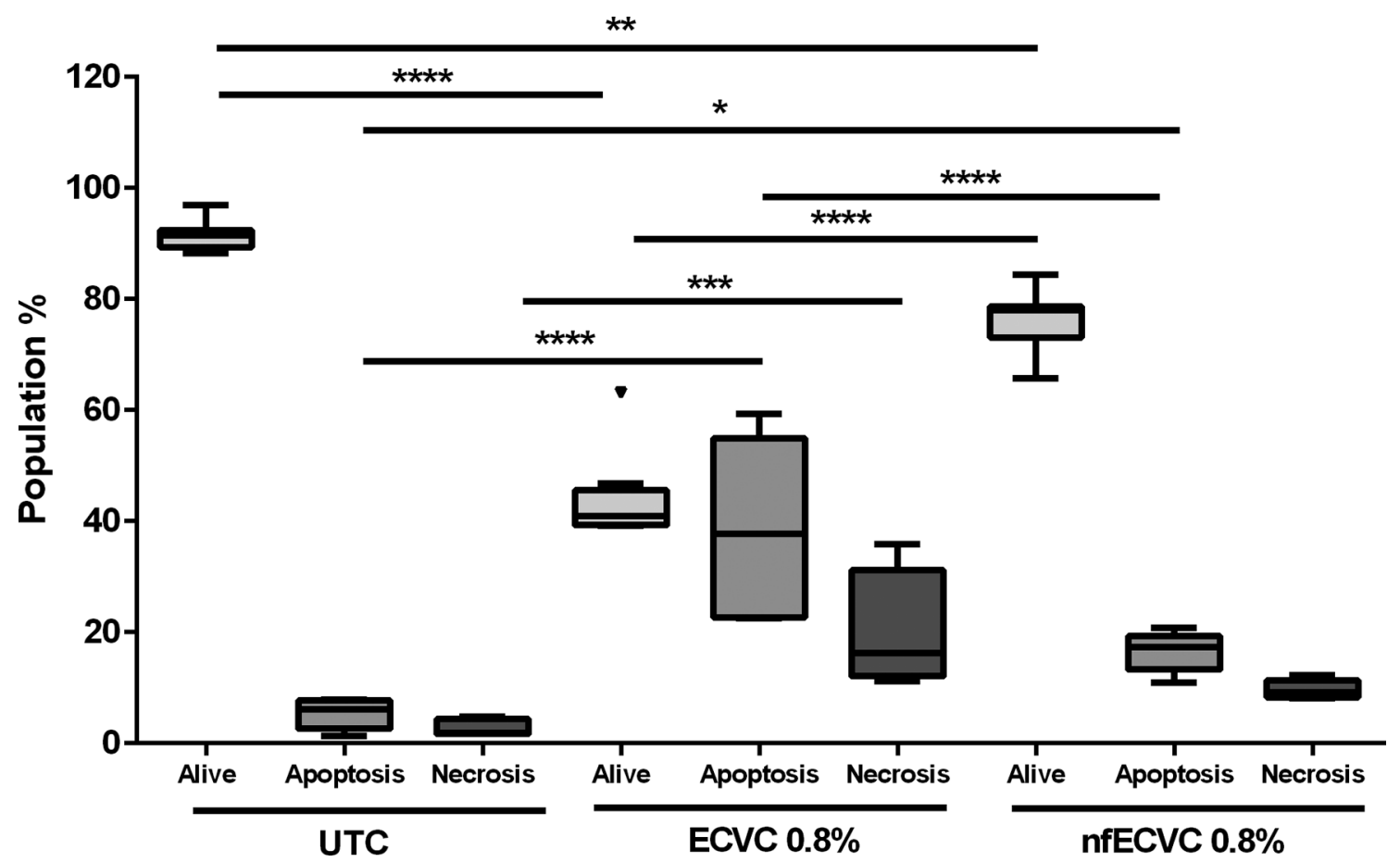

Figure 2 Effect of e-cigarette vapour condensate (ECVC)/nicotine-free ECVC (nfECVC) on alveolar macrophage (AM) apoptosis and necrosis. Induction of apoptosis and necrosis in AM following a 24 hour exposure to $0.8 \%$ ECVC/nfECVC. Graphs presented as median with IQR of eight independent experiments. The central horizontal line on each box plot represents the median, the upper and lower horizontal lines represent the first (Q1) and third (Q3) quartiles, respectively, and the vertical lines represent the range of values within the limits Q1-1.5 (Q3-Q1) and Q3-1.5 (Q3-Q1). $\mathrm{n}=8,{ }^{*} \mathrm{P}<0.05$, ${ }^{*} \mathrm{P}<0.01,{ }^{* *} \mathrm{P}<0.001,{ }^{* * *} \mathrm{P}<0.0001$. UTC, untreated control.

Contrastingly ECVC/nfECVC produced a greater reduction in viability following a $0.8 \%(\mathrm{v} / \mathrm{v})$ challenge (figure $1 \mathrm{C}, \mathrm{D})$ : ECVC: $18.2 \%$ viable (IQR $15.7 \%-19.5 \%, \mathrm{P}<0.001$ ), nfECVC $62.8 \%$ viable (IQR 49.9\%-68.1\%p<0.0001) compared with UTC. Viability of AMs was maintained better in the nicotine-free challenge (nfECL/nfECVC), than that containing nicotine (figure $1 \mathrm{~A} / \mathrm{C}$ vs $\mathrm{B} / \mathrm{D}$ ), suggesting that although vaping significantly increases the cytotoxic potential of ECL, much of the cytotoxic effect was nicotine dependent.

\section{ECVC induces AM apoptosis and necrosis and is exaggerated} by the presence of nicotine

AMs were exposed to $0.8 \%$ ECVC/nfECVC for 24 hours and compared with UTC. After 24 hours the majority of cells remained viable; median: 92.5\% (IQR 91.5\%-96.9\%, (Annexin-/Pi-), with low levels of apoptosis (Annexin + cells): $6.17 \%$ (IQR 2.63\%-7.77\%), and necrosis (Annexin-/PI+): 1.9\% (IQR 1.7\%-4.4\%) (figure 2).

After 24 hours, total viable cells were significantly reduced when treated with either ECVC (40.87\% alive, IQR 39.29\%$45.61 \%, \mathrm{P}<0.0001)$ or nfECVC $(77.94 \%$ alive, IQR $73.09 \%-$ $78.69 \%, \mathrm{P}<0.01)$ compared with UTC.

ECVC exposure significantly increased apoptosis $(37.7 \%$, IQR 22.7\%-54.9\%, P<0.0001) and necrosis (16.3\%, IQR $12.1 \%-31.2 \%, \mathrm{P}<0.001)$ compared with controls. Exposure to nfECVC also increased apoptosis significantly (17.36, IQR 13.28\%-19.4\%, P<0.05), but not necrosis $(9.27 \%$, IQR $8.3 \%-11.3 \%$ )

ECVC treatment induced significantly more apoptosis than nfECVC (17.4\%, IQR 13.3\%-19.4\%, P<0.0001) and resulted in a greater total loss of viable cells after 24 hours of treatment $(\mathrm{P}<0.0001)$. These data confirm the cytotoxic effects of ECVC and support both a nicotine-dependent and nicotine-independent effect.

\section{Effect of ECVC on macrophage function}

The effects of sub-lethal ECVC exposure were assessed using measures of macrophage function important in the innate immune response, namely ROS production, cytokine, chemokine and protease release, as well as bacterial (Escherichia coli and Staphylococcus aureus) phagocytosis.

\section{ROS are induced by ECVC treatment}

ROS production was assessed following exposure of AMs to a sub-cytotoxic dose $(0.5 \%)$ of ECVC/nfECVC for 4 hours. Untreated macrophages showed a low baseline level of ROS production (figure 3) (1085, IQR 863.7-1133 relative fluorescence units (RFUs)). Condensate challenge resulted in a 50-fold increase in ROS production for both ECVC (53 858, IQR 48375-56 425 RFU, P<0.0001) and nfECVC (48 746, IQR 44 238-56 063 RFU, $\mathrm{P}<0.0001$ ) compared with UTC.

\section{Pro-inflammatory cytokines, chemokines and proteases are induced by 24 hour exposure to $0.5 \%$ ECVC}

The effects on pro-inflammatory cytokines, chemokines and metalloprotease production is shown in figure 4. $0.5 \%$ ECVC significantly induced production of all analytes: IL-6 $(\mathrm{P}<000.1)$, TNF- $\alpha(\mathrm{P}<0.001)$, CXCL8 $(\mathrm{P}<0.0001)$, MCP-1 $(\mathrm{P}<0.01)$ and matrix metalloprotease $9(\mathrm{MMP}-9)(\mathrm{P}<0.0001)$ compared with UTC. The response to nfECVC was more variable, with a lower increase in IL-6 $(\mathrm{P}<0.001)$, CXCL-8 $(\mathrm{P}<0.0001)$ and MMP-9 $(\mathrm{P}<0.0001)$ compared with UTC. Non-significant changes were also seen for TNF- $\alpha$ and MCP-1. 


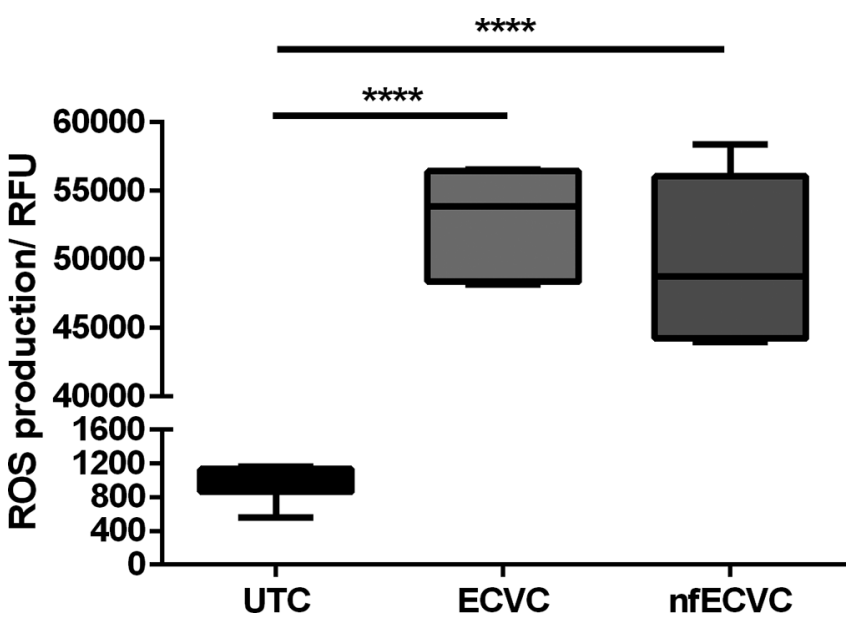

Figure 3 Functional effects of e-cigarette vapour condensate (ECVC)/ nicotine-free ECVC (nfECVC) exposure to alveloar macrophages (AMs) on reactive oxygen species (ROS). AMs were exposed to $0.5 \% \mathrm{ECVCI}$ nfECVC for 4 hours. Following this, production of ROS was assessed by DCFDA assay. Graphs presented as median with IQR of eight independent experiments. The central horizontal line on each box plot represents the median, the upper and lower horizontal lines represent the first (Q1) and third (Q3) quartiles, respectively, and the vertical lines represent the range of values within the limits Q1-1.5 (Q3-Q1) and Q3-1.5 (Q3-Q1). $\mathrm{n}=8,{ }^{*} \mathrm{P}<0.05$, ${ }^{*} \mathrm{P}<0.01$, ${ }^{*}{ }^{*} \mathrm{P}<0.001$, $* * * * P<0.0001$.

\section{ECVC significantly inhibits phagocytosis by AM and THP-1} macrophages

Incubation of AMs with ECVC and nfECVC reduced pHrodo E. coli BioParticle phagocytosis by $30 \%(\mathrm{P}<0.0001)$ and $50.2 \%$ respectively $(\mathrm{P}<0.0001$, figure $5 \mathrm{~A})$.

Incubation of THP-1 macrophages with ECVC and nfECVC reduced pHrodo E. coli BioParticle phagocytosis by $41.7 \%$ $(\mathrm{P}<0.0001)$ and $48.5 \%$ respectively $(\mathrm{P}<0.0001$, figure $5 \mathrm{~B})$.
Incubation of THP-1 macrophages with ECVC and nfECVC reduced pHrodo $S$. aureus BioParticles phagocytosis by $60.9 \%$ $(\mathrm{P}<0.0001)$ and $62.9 \%$ respectively $(\mathrm{P}<0.0001$, online supplementary figure E4).

\section{The effect of $\mathrm{N}$-acetyl cysteine treatment following ECVC challenge}

ROS production in response to cigarette smoking (or smoke extract) has been implicated as a mediator of adverse effects, ${ }^{23}$ therefore we examined the possible utility of $\mathrm{N}$-acetyl cysteine (NAC) treatment in reducing the harmful effects of ECVC in THP-1 macrophages. Both AM and THP-1 macrophages were used for these experiments due to the large number of experimental conditions and numbers of cells required.

\section{NAC can ameliorate the cytotoxic effects of ECVC on THP-1} derived macrophages

Similar to our observations in AMs, ECVC and nfECVC were cytotoxic to THP-1-derived macrophages $(24.4 \%$, IQR 21.8\%-27.4\%, P<0.0001; 62.8\%, IQR 54.4\%-68.4\%, $\mathrm{P}<0.0001$, respectively(. $1 \mathrm{mM}$ NAC treatment given simultaneously with condensate challenge prevented the effects on viability of both ECVC and nfECVC compared with UTC (figure 6A).

\section{NAC can ameliorate the pro-apoptotic effects of ECVC on THP-1-derived macrophages}

ECVC challenge of THP-1 macrophages increased apoptosis to 36.1\% (IQR 35.8\%-39.8\%, P<0.0001, figure 5B). Exposure to nfECVC increased apoptosis to a lesser degree (18.8\%, IQR 13.8\%-20.2\%, $\mathrm{P}<0.0001$, figure 6B). NAC treatment given simultaneously with condensate challenge significantly reduced apoptosis in both ECVC (5.9\%, IQR 4.6\%-7.4\%, P<0.0001) and nfECVC $(4.5 \%$, IQR $2.4 \%-4.6 \%, \mathrm{P}<0.0001)$ challenged cells (figure $5 \mathrm{~B}$ ).
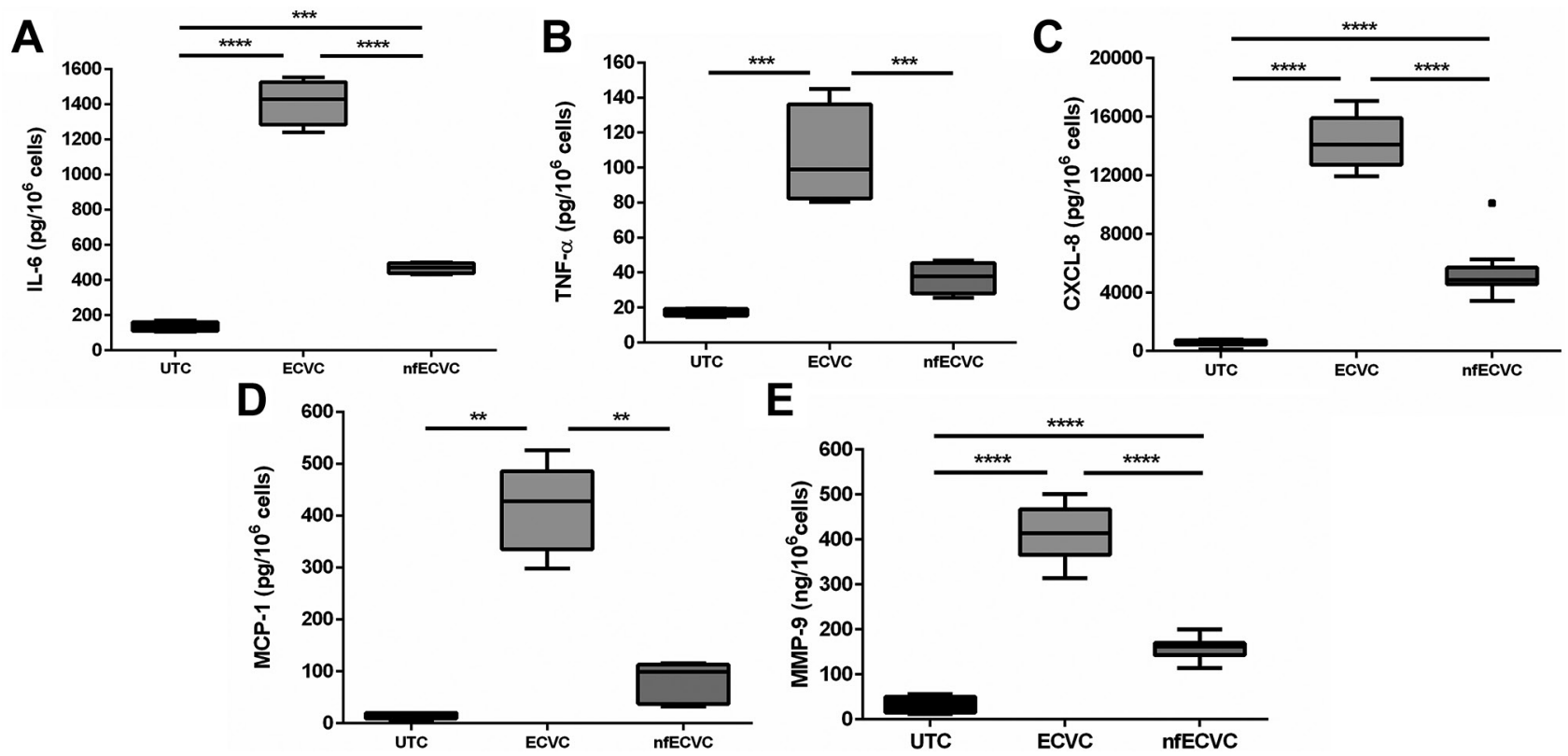

Figure 4 Functional effects of e-cigarette vapour conensate (ECVC)/nicotine-free ECVC (nfECVC) exposure to alveolar macrophages (AMs). Production of inflammatory cytokines $(A, B)$, chemokines $(C, D)$ and $(E)$ matrix metalloproteinase (MMP-9). AMs following 24 hour exposure to ECVC $(0.5 \%)$ as assessed by ELISA. Data are presented as pg/106 live cells at the end of the experiment to account for cell loss. $n=8,{ }^{*} P<0.05,{ }^{*} P<0.01$, $* * * \mathrm{P}<0.001, * * * * \mathrm{P}<0.0001$ 
A

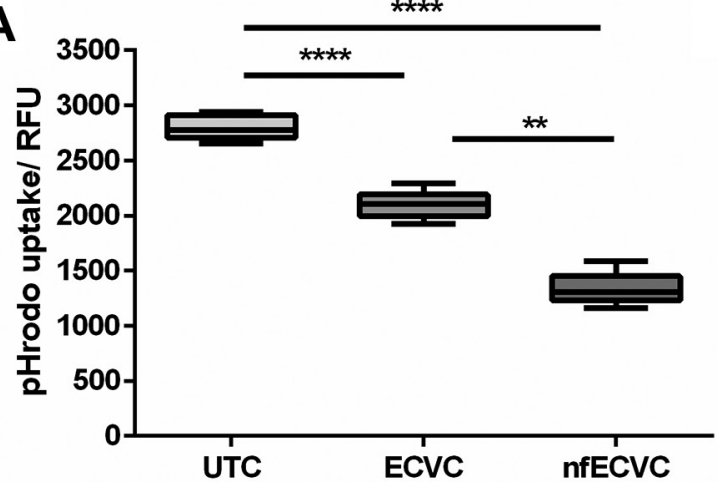

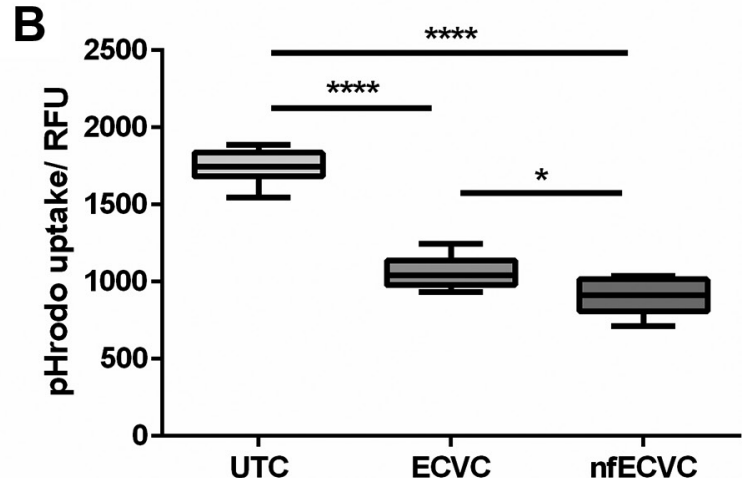

Figure 5 Functional effects of e-cigarette vapour condensate (ECVC)/nicotine-free ECVC (nfECVC) exposure to (A) alveolar macrophage (AM) and (B) THP-1 macrophage phagocytosis. Cells were exposed to $0.5 \%$ ECVC/nfECVC for 6 hours, following which uptake of pHrodo bioparticles was assessed. Graphs presented as median with IQR of eight independent experiments. The central horizontal line on each box plot represents the median, the upper and lower horizontal lines represent the first (Q1) and third (Q3) quartiles, respectively, and the vertical lines represent the range of values within the limits $\mathrm{Q} 1-1.5$ (Q3-Q1) and Q3-1.5 (Q3-Q1). $\mathrm{n}=8$, ${ }^{*} \mathrm{P}<0.05,{ }^{*} \mathrm{P}<0.01,{ }^{* *}{ }^{*} \mathrm{P}<0.001,{ }^{* * * *} \mathrm{P}<0.0001$. UTC, untreated control.

\section{NAC can restore phagocytic function of ECVC-treated macrophages}

Incubation of AMs with ECVC and nfECVC reduced pHrodo E. coli BioParticle phagocytosis (figure $6 \mathrm{~A})$. NAC $(1 \mathrm{mM})$ given simultaneously with ECVC/nfECVC restored phagocytic function $(\mathrm{P}<0.001$ ), although not to pretreatment levels (figure $7 \mathrm{~A}$ ).

Using THP-1 macrophages, an increased dose of NAC treatment was assessed to determine if a greater protective effect could be achieved. ECVC treatment reduced phagocytosis of $E$. coli pHrodo BioParticles by $41.9 \%$ (figure $6 \mathrm{~B}, \mathrm{P}<0.0001$ ) and nfECVC by $48.4 \%$ (figure $7 \mathrm{~B}, \mathrm{P}<0.0001$ ). Simultaneous treatment with NAC $(5 \mathrm{mM})$ restored phagocytic function to levels comparable to the control (figure $6 \mathrm{~B}, \mathrm{P}<0.0001$ ). Higher doses of NAC had an even greater restorative effect on cell function.

Phagocytosis of S. aureus pHrodo bioparticles was also significantly restored by simultaneous NAC treatment. (Online supplementary figure E4)

\section{ECVC effects on THP-1 macrophage viability and apoptosis} are attenuated by inhibitors of phosphopinositol 3 kinase ROS-induced lung inflammation in COPD has been reported to be associated with phosphopinositol 3 kinase (PI3K) activation. ${ }^{24}$
To explore a role for PI3K in ECVC-induced responses we used the pan- inhibitor LY294002 $(5 \mathrm{nM})$ as well as an isoform selective inhibitor (PIK75 $10 \mathrm{nM}$ ).

Both general PI3K inhibition (online supplementary figure E5a) and PI3K $\alpha$ isoform inhibitor (PIK-75, figure 8 ) attenuated the effects of ECVC (Ly294002; 37.4\%, PIK75; 35\% increase in viability compared with ECVC, $\mathrm{P}<0.0001)$. This protective effect was also evident when cells were challenged with nfECVC (Ly294002 25\%, PIK75 29.2\% increase in viability compared with nfECVC, $\mathrm{P}<0.0001)$. PI3K inhibition was also shown to partially restore phagocytic capacity (online supplementary figure E6), after challenge with sub-cytotoxic levels of both ECVC (Ly294002; 21.3\%, PIK75; 23.2\% restoration compared with ECVC alone, $\mathrm{P}<0.005)$ and nfECVC (Ly294002; 25.8\%, PIK75; 20.9\% compared with nfECVC alone, $\mathrm{P}<0.005)$.

\section{DISCUSSION}

We have validated a simple, cheap and effective system for condensing vaped ECL vapour to enable in vitro work. This is the first study to report human AM responses to ECVC and demonstrates dose-dependent cytotoxicity, inducing apoptosis with both nicotine dependent and independent responses
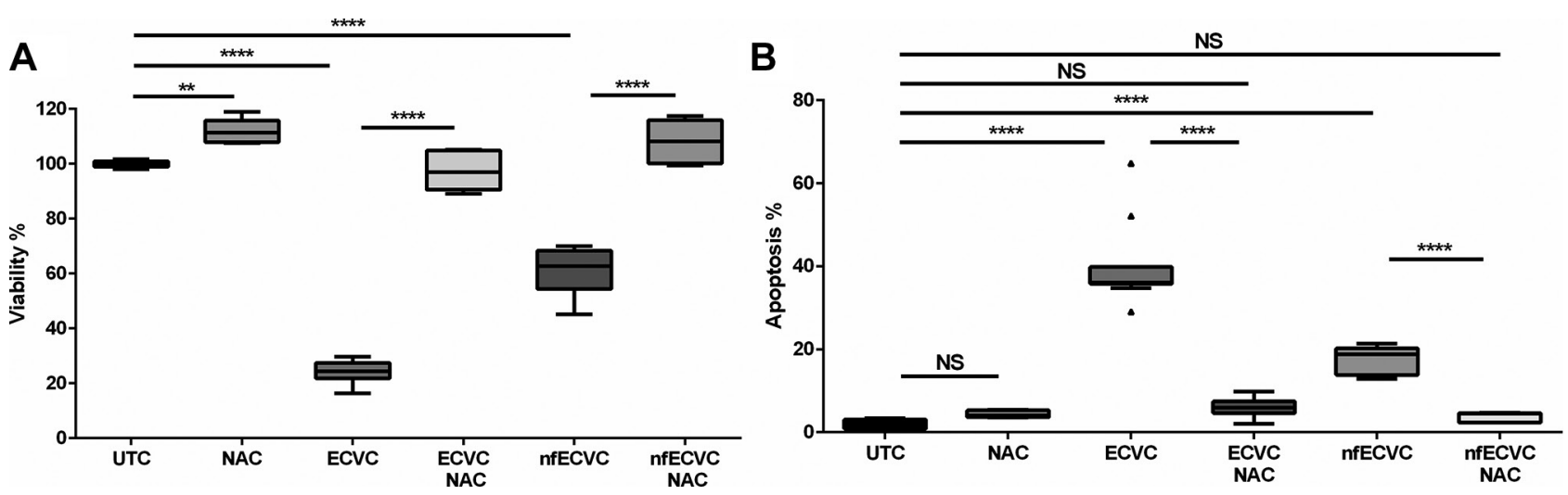

Figure 6 Effect of antioxidant treatment on macrophages. (A) Viability following 24hour exposure to e-cigarette vapour condensate (ECVC)/ nicotine-free vapour condensate (nfECVC) in the presence or absence of $\mathrm{N}$-acetyl cysteine (NAC). Graphs presented as median with IQR of eight independent experiments. (B) Apoptosis following 24 hour exposure to ECVC/nfECVC, in the presence or absence of NAC. Graphs presented as median with IQR of six independent experiments. The central horizontal line on each box plot represents the median, the upper and lower horizontal lines represent the first (Q1) and third (Q3) quartiles, respectively, and the vertical lines represent the range of values within the limits Q1-1.5 (Q3-Q1) and Q3-1.5 (Q3-Q1). $n=8,{ }^{*} \mathrm{P}<0.05,{ }^{* *} \mathrm{P}<0.01,{ }^{* *} \mathrm{P}<0.001,{ }^{* * * *} \mathrm{P}<0.0001$. UTC, untreated control. 

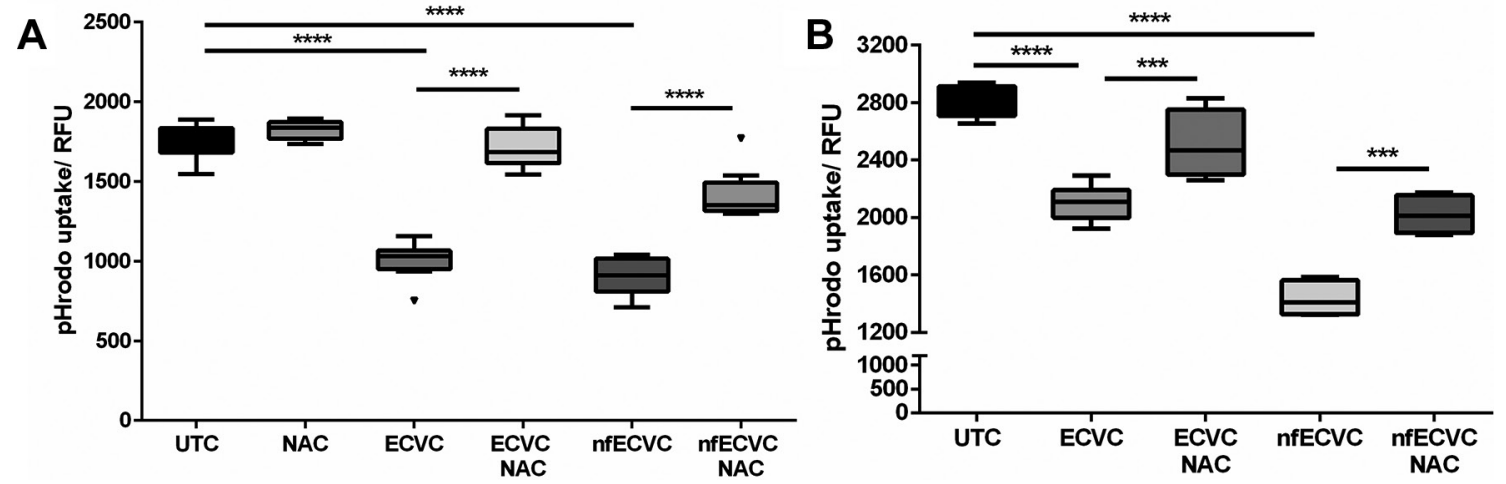

Figure 7 Functional effects of e-cigarette vapour condensate (ECVC)/nicotine-free vapour condensate (nfECVC) phagocytosis. (A) Alveolar macrophages (AMs) exposed to $0.5 \%$ ECVC/nfECVC for 6 hours in the presence or absence of $1 \mathrm{mM}$ NAC treatment, following which uptake of pHrodo bioparticles was assessed. (B) THP-1 macrophages were exposed to $0.5 \%$ ECVC/nfECVC for 6 hours, in the presence or absence of $5 \mathrm{mM} \mathrm{NAC}$ treatment, following which uptake of pHrodo bioparticles was assessed. Graphs presented as median with IQR of eight independent experiments. The central horizontal line on each box plot represents the median, the upper and lower horizontal lines represent the first (Q1) and third (Q3) quartiles, respectively, and the vertical lines represent the range of values within the limits Q1-1.5 (Q3-Q1) and Q3-1.5 (Q3-Q1). $n=8,{ }^{*} \mathrm{P}<0.05,{ }^{*} \mathrm{P}<0.01$,

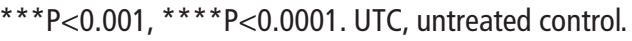

which the vaping process accentuates. At sub-cytotoxic doses, ECVC enhances production of ROS, inflammatory cytokines, chemokines and metalloproteinases, although the response is less pronounced with nfECVC. Bacterial phagocytosis by macrophages is inhibited acutely by ECVC and the effects are attenuated by the anti-oxidant NAC, suggesting ROS and reactive aldehydes play a role in the effects of ECVC/nfECVC. These effects appear to be partially PI3K dependent.

We have confirmed that vaping exaggerates the cytotoxic effects of ECL, inducing both cellular apoptosis and necrosis. These effects were seen when AMs were treated with both ECVC and nfECVC, suggesting both nicotine dependent and independent mechanisms.
Several studies have examined the change in composition of e-cigarette vapour, ${ }^{2} 3^{7-11}$ and have identified many different chemicals that could be toxic, including free radicals, particulates, formaldehyde, nitrosamines, volatile organic compounds and polycyclic aromatic hydrocarbons. ${ }^{3} 8$ Importantly, the levels of several of these toxicants have been reported to be increased after vapourisation, due to heat and/orvoltage generated by the battery in e-cigarettes. ${ }^{2}{ }^{7-11}$ Many variables affect constituents of the vapour produced: the nicotine content, the ratio of humectants PG and VG present, the energy input used in the vapourising process, and the temperature achieved are all important factors.

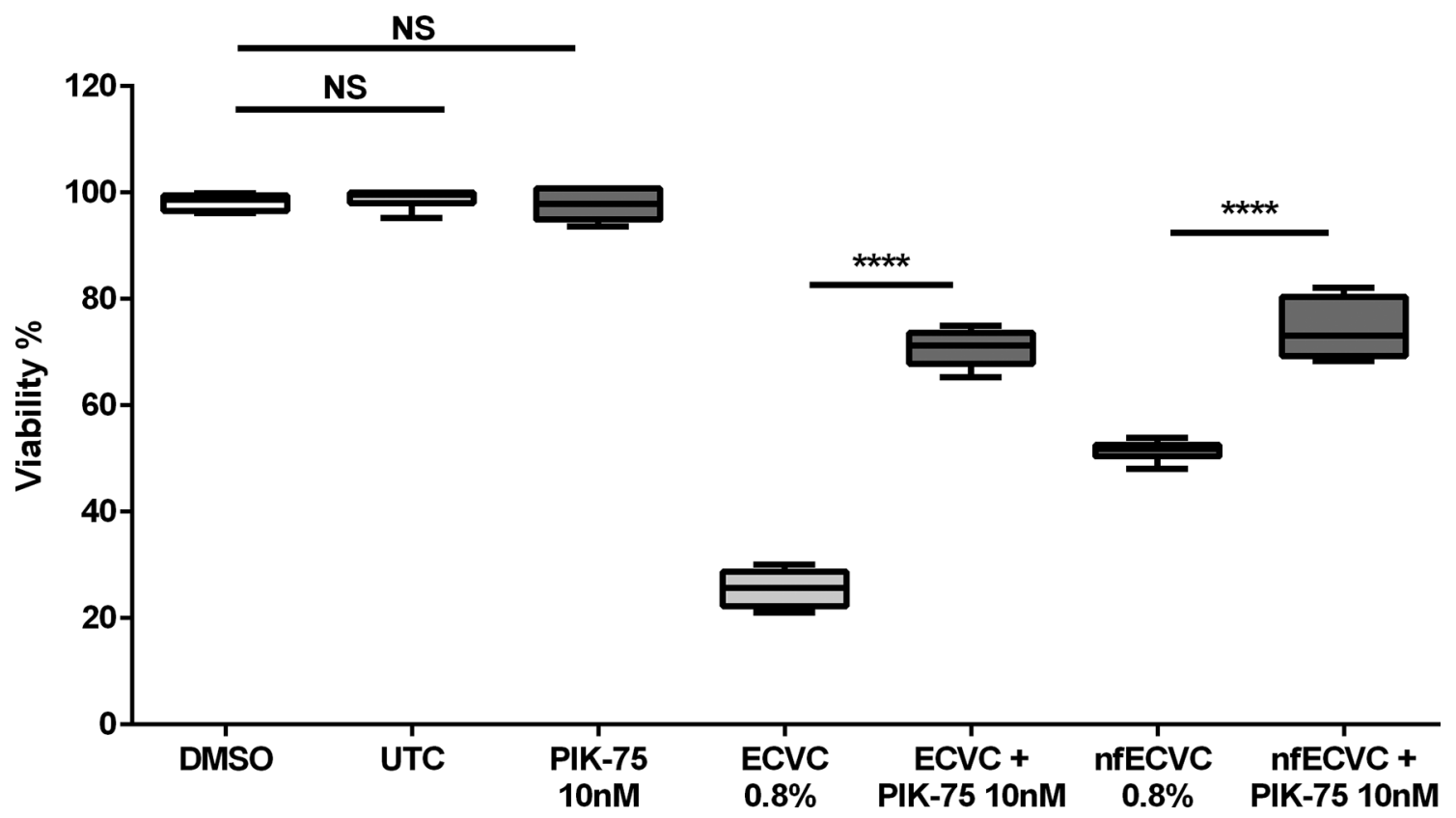

Figure 8 Effect of e-cigarette vapour condensate (ECVC)/nicotine-free ECVC (nfECVC) and phosphopinositol 3 kinase (PI3K) inhibitor PIK-75 on alveolar macrophage viability. Viability was assessed by 4 hour incubation with cell titre aqueous assay following 24 hour exposure to class one specific PI3K inhibitor PIK-75 and challenged with ECVC or nfECVC (0.8\%). Graphs presented as median with IQR of six independent experiments. The central horizontal line on each box plot represents the median, the upper and lower horizontal lines represent the first (Q1) and third (Q3) quartiles, respectively, and the vertical lines represent the range of values within the limits $\mathrm{Q} 1-1.5(\mathrm{Q} 3-\mathrm{Q} 1)$ and $\mathrm{Q} 3-1.5(\mathrm{Q} 3-\mathrm{Q} 1) . \mathrm{n}=8$, ${ }^{*} \mathrm{P}<0.05,{ }^{*} \mathrm{P}<0.01$, ${ }^{* * *} \mathrm{P}<0.001,{ }^{* * *} \mathrm{P}<0.0001$. UTC, untreated control. 
E-cigarette vapour has been reported to contain up to $7 \times 10^{11}$ free radicals per puff. ${ }^{10}$ Both nicotine-free and nicotine-containing condensate induced a significant increase in ROS release from our AMs, which may explain the induction of apoptosis in nicotine-free liquid. There was significantly greater ROS production in AMs treated with nicotine-containing condensate than in nicotine-free condensate, once again suggesting both nicotine dependent and independent mechanisms at work. Consistent with our results, nicotine has been shown to increase ROS production in both epithelial cells ${ }^{25}$ and macrophages. ${ }^{26} \mathrm{~A}$ recent patient study by Reidel et al examined the protein content of induced sputum in e-cigarette users and smokers. ${ }^{27}$ In support of our findings, proteomic analysis showed significant upregulation of oxidative stress-related proteins in both smokers and vapers, such as MMP-9, known to be implicated in inflammatory lung diseases such as COPD. The effects of ECVC with and without were ameliorated by NAC and PI3K inhibition.

Sub-lethal exposure of AMs to ECVC induced significantly more cytokine, chemokine and MMP-9 production than nfECVC. Others have similarly reported a significant induction of IL-6 and CXCL-8 in H292 cells following exposure to ECL but not following nfECL challenge. ${ }^{28}$ The importance of nicotine in ECL is reflected in the lesser effect on cytokine release and was also recently confirmed in vivo in a murine aerosol model using unvaped fluid. ${ }^{5}$

Detection and phagocytosis of pathogens is key to macrophage function and in many cases is the first step in orchestrating an immune response to infection in the airways. Any effect of e-cigarette vapour on the phagocytic ability of AMs is therefore of potential significance to the innate immune response in vivo. At sub-cytotoxic levels both ECVC and nfECVC inhibited phagocytosis of E. coli and $S$. aureus, suggesting vaping might significantly impair bacterial clearance. Our data are supported by murine models in which mice exposed to e-cigarette vapour showed significantly impaired pulmonary bacterial clearance compared with air-exposed mice following an intranasal infection with Streptococcus pneumoniae. This defective bacterial clearance was due to reduced phagocytosis by AMs from e-cigarette vapour exposed mice. ${ }^{29}$ A recent human volunteer study $^{30}$ found e-cigarette vapour significantly increased platelet-activating factor receptor (PAFR) expression, which aids pneumococcal adhesion to airway cells. In vitro PAFR is significantly upregulated by inducers of oxidative stress such as traditional cigarette smoke. Miyashita et al demonstrated increased PAFR leads to increased pneumococcal adhesion. ${ }^{30}$ A broader study also found risk of bronchitic symptoms was increased by almost twofold among e-cigarette users. ${ }^{31}$ These studies in human volunteers support our suggestion that e-cigarette usage may lead to increased or more serious respiratory tract infections, however further community-based studies will be required to fully assess the effect on lung health of e-cigarette users.

Interestingly, in these experiments nfECVC produced a greater inhibitory effect than ECVC on phagocytosis. Activation of nicotinic acetylcholine receptors ( $\mathrm{AAChR}$ ) has been shown to upregulate phagocytosis in tissue-resident macrophages. ${ }^{32} \mathrm{Nico}-$ tine may therefore be offsetting some of the inhibitory effects in both ECVC and nfECVC, which are working through a nicotine-independent mechanism.

Cytotoxic compounds generated during the vaping process, ${ }^{3} 8$ such as reactive aldehyde species-formaldehyde, acetyl aldehyde and acrolein-are known to induce apoptosis by lipid peroxidation. ${ }^{33}$ Reactive aldehydes further cause the accumulation of 4-hydroxynonenal (4-HNE) which can induce apoptosis via the Fas-mediated and P53-dependent pathways.
HNE formation can also be caused by inflammation-induced ROS. $^{3435}$ With this in mind, we performed experiments using THP-1-derived macrophages challenged with a cytotoxic dose $(0.8 \%)$ of ECVC/nfECVC in the presence and absence of NAC, a well characterised anti-oxidant and anti-aldehyde. NAC significantly attenuated both the cytotoxic activity and pro-apoptotic effects of condensate with or without nicotine. As shown with AMs, in THP-1 macrophages, ECVC caused significantly greater loss of viability and significantly more apoptosis than nfECVC, again suggesting a nicotine dependent and independent mechanism of action.

Traditional cigarette smoking is implicated as the cause of COPD in at least $20 \%$ of smokers, which is characterised by increased neutrophilic inflammation and oxidative stress within the lung. ${ }^{24} 3637$ The effects of oxidative stress in epithelial cells are mediated through micro-RNA34a via activation of PI3K $\alpha^{24}$ Micro-RNA34a has been implicated in accelerated cellular senescence, inducing a proliferative, apoptotic phenotype. These effects were aborogated by use of PI3K inhibitors restoring the baseline phenotype. ${ }^{24}$ Nicotine receptor seven is highly expressed on alveolar macrophages and its activation has also been shown to activate PI3K. ${ }^{38} 39$ We therefore examined the effects of pan PI3K inhibitor Ly294002 and class one isoform selective inhibitor PIK-75 on THP-1-derived macrophages. There was a significant protective effect after PI3K inhibition with Ly294002. This effect was mostly conserved when class one isoform inhibitor PIK-75 was used. PIK-75 selectively inhibits the $\mathrm{p} 110 \alpha$ subunit 200 -fold more potently than $\mathrm{p} 110 \beta$ subunit, suggesting a large portion of the activity is moderated through the class one isoform. However, further work remains to be carried out to fully elucidate the mechanism(s) of action of ECVC/nfECVC on AMs.

This study has limitations. First, we have used an in vitro study on primary AMs with exposure levels which may not be physiological but are more reflective of the inhalant. In addition, the nicotine-containing ECL we selected has been shown to induce changes reflective of COPD in mice, suggesting the results have physiological plausibility. Second it is difficult to determine an optimal dose of nicotine exposure. In this study we have used $0.8 \%$ ECVC (containing $208 \mu \mathrm{g} / \mathrm{mL}$ nicotine) and $0.5 \%$ ECVC (containing $130 \mu \mathrm{g} / \mathrm{mL}$ nicotine). It is not possible to determine a standard nicotine dose for smoking experiments as each individual will titrate their nicotine intake to match their requirement. An average cigarette has $10-14 \mathrm{mg}$ of nicotine, ${ }^{40}$ and while the majority of this nicotine is not absorbed, intake is approximately $1-1.5 \mathrm{mg} .{ }^{41}$ Puffing topography studies of e-cigarette users ${ }^{1620}$ have shown nicotine intake of approximately $1.2 \mathrm{mg}$ in a $20 \mathrm{~min}$ vaping session, with users also titrating intake to maintain their specific plasma nicotine concentration. There is a lack of information about epithelial lining fluid levels of nicotine in smokers. AM exposure in vivo will also vary according to techniques used by users to modify their nicotine intake, such as depth and frequency of intake as well as breath-holding/expiration. ${ }^{162042}$

Third, our model represents an acute exposure, rather than a chronic exposure system which is better suited to in vivo animal experiments. Fourth, there is currently a huge disparity in the literature regarding e-cigarettes. Many groups have championed the benign nature of ECL while others have shown the cytotoxic effects of ECL in vitro and in vivo. This disparity may reflect the lack of a standardised model of in vitro cellular exposure and interpretation. Therefore, until a gold standard is established, continued controversy is likely. However, our model seeks to replicate the actual exposure of the users' AMs post vaping. We believe this is an important step in establishing an in vitro system 
by which to investigate the effects of e-cigarettes on the airways. Finally, we have not assessed the effects of flavours on cellular effects, ${ }^{43}$ partly because this adds another layer of uncertainty and potential confounders. However, the data provide a background on which to study these other potential factors, with and without nicotine.

In conclusion, we sought to replicate the potential effects of exposure of the user in an acute in vitro system using our vaping-condensate technique. We show a significant increase in cytotoxicity caused by the vaping process itself. Importantly, exposure of macrophages to ECVC induced many of the same cellular and functional changes in AM function seen in cigarette smokers and patients with COPD. While further research is needed to fully understand the effects of e-cigarette exposure in humans in vivo, we suggest continued caution against the widely held opinion that e-cigarettes are safe.

Contributors Author Contributions: Concept and design: DRT, AS, STL, DD, KEL, BN, RF. Cell preparation, laboratory work and data analysis: AS, RM, STL, KA. GCFIDAB. Additional laboratory work: FSG. Patient recruitment: BN, DRT, DP, RM. Drafting manuscript: AS, DRT. ALL authors have read and approved the manuscript.

Funding DRT (G1100196/1), ES (MR/L008335/1), AS (MR/L002736/1) and RM (MR/N021185/1) were funded by the MRC. AS, STL and DD were supported by the British Lung Foundation (PPRG16-12).

Competing interests None declared.

Patient consent Not required.

Provenance and peer review Not commissioned; externally peer reviewed.

Open access This is an open access article distributed in accordance with the Creative Commons Attribution Non Commercial (CC BY-NC 4.0) license, which permits others to distribute, remix, adapt, build upon this work non-commercially, and license their derivative works on different terms, provided the original work is properly cited, appropriate credit is given, any changes made indicated, and the use is non-commercial. See:http://creativecommons.org/licenses/by-nc/4.0/.

\section{REFERENCES}

1 Rom 0, Pecorelli A, Valacchi G, et al. Are E-cigarettes a safe and good alternative to cigarette smoking? Ann N Y Acad Sci 2015;1340:65-74.

2 Higham A, Rattray NJ, Dewhurst JA, et al. Electronic cigarette exposure triggers neutrophil inflammatory responses. Respir Res 2016;17:56.

3 Chun LF, Moazed F, Calfee CS, et al. Pulmonary toxicity of e-cigarettes. Am J Physiol Lung Cell Mol Physiol 2017;313:L193-L206.

4 Lerner CA, Sundar IK, Watson RM, et al. Environmental health hazards of e-cigarettes and their components: Oxidants and copper in e-cigarette aerosols. Environ Pollut 2015;198:100-7.

5 Williams M, Villarreal A, Bozhilov K, et al. Metal and silicate particles including nanoparticles are present in electronic cigarette cartomizer fluid and aerosol. PLOS One 2013;8:e57987.

6 Mikheev VB, Brinkman MC, Granville CA, et al. Real-Time Measurement of Electronic Cigarette Aerosol Size Distribution and Metals Content Analysis. Nicotine Tob Res 2016;18:1895-902.

7 Geiss 0, Bianchi I, Barrero-Moreno J. Correlation of volatile carbonyl yields emitted by e-cigarettes with the temperature of the heating coil and the perceived sensorial quality of the generated vapours. Int J Hyg Environ Health 2016;219:268-77.

8 Kosmider L, Sobczak A, Fik M, et al. Carbonyl compounds in electronic cigarette vapors: effects of nicotine solvent and battery output voltage. Nicotine Tob Res 2014;16:1319-26

9 Kosmider L, Sobczak A, Prokopowicz A, et al. Cherry-flavoured electronic cigarettes expose users to the inhalation irritant, benzaldehyde. Thorax 2016;71:376-7.

10 Leigh NJ, Lawton Rl, Hershberger PA, et al. Flavourings significantly affect inhalation toxicity of aerosol generated from electronic nicotine delivery systems (ENDS). Tob Control 2016;25(Suppl 2):ii81-ii87.

11 Margham J, McAdam K, Forster M, et al. Chemical Composition of Aerosol from an E-Cigarette: A Quantitative Comparison with Cigarette Smoke. Chem Res Toxicol 2016;29:1662-78.

12 Aug A, Altraja S, Kilk K, et al. E-Cigarette Affects the Metabolome of Primary Normal Human Bronchial Epithelial Cells. PLoS One 2015;10:e0142053.

13 Garcia-Arcos I, Geraghty P, Baumlin N, et al. Chronic electronic cigarette exposure in mice induces features of COPD in a nicotine-dependent manner. Thorax 2016;71:1119-29.
14 Lerner CA, Sundar IK, Yao H, et al. Vapors produced by electronic cigarettes and e-juices with flavorings induce toxicity, oxidative stress, and inflammatory response in lung epithelial cells and in mouse lung. PLoS One 2015;10:e0116732.

15 Vlahos R, Bozinovski S. Role of alveolar macrophages in chronic obstructive pulmonary disease. Front Immunol 2014;5:435.

16 Behar RZ, Hua M, Talbot P. Puffing topography and nicotine intake of electronic cigarette users. PLOS One 2015;10:e0117222.

17 Farsalinos KE, Spyrou A, Tsimopoulou K, et al. Nicotine absorption from electronic cigarette use: comparison between first and new-generation devices. Sci Rep 2014:4:4133.

18 Goniewicz ML, Knysak J, Gawron M, et al. Levels of selected carcinogens and toxicants in vapour from electronic cigarettes. Tob Control 2014;23:133-9.

19 Hua M, Yip H, Talbot P. Mining data on usage of electronic nicotine delivery systems (ENDS) from YouTube videos. Tob Control 2013;22:103-6.

20 Robinson RJ, Hensel EC, Morabito PN, et al. Electronic Cigarette Topography in the Natural Environment. PLoS One 2015;10:e0129296.

21 Norton KJ, June KM, O'Connor RJ. Initial puffing behaviors and subjective responses differ between an electronic nicotine delivery system and traditional cigarettes. Tob Induc Dis 2014;12:17.

22 Tsuchiya S, Kobayashi Y, Goto Y, et al. Induction of maturation in cultured human monocytic leukemia cells by a phorbol diester. Cancer Res 1982;42:1530-6.

23 Anderson C, Majeste A, Hanus J, et al. E-Cigarette Aerosol Exposure Induces Reactive Oxygen Species, DNA Damage, and Cell Death in Vascular Endothelial Cells. Toxicol Sci 2016;154:332-40.

24 Baker JR, Vuppusetty C, Colley T, et al. Oxidative stress dependent microRNA-34a activation via PI3K $\alpha$ reduces the expression of sirtuin-1 and sirtuin-6 in epithelial cells. Sci Rep 2016;6:35871.

25 Wetscher GJ, Bagchi D, Perdikis G, et al. In vitro free radical production in rat esophageal mucosa induced by nicotine. Dig Dis Sci 1995;40:853-8.

26 Mahapatra SK, Das S, Bhattacharjee S, et al. In vitro nicotine-induced oxidative stress in mice peritoneal macrophages: a dose-dependent approach. Toxicol Mech Methods 2009;19:100-8.

27 Reidel B, Radicioni G, Clapp PW, et al. E-Cigarette Use Causes a Unique Innate Immune Response in the Lung, Involving Increased Neutrophilic Activation and Altered Mucin Secretion. Am J Respir Crit Care Med 2018;197:492-501.

28 Gerloff J, Sundar IK, Freter R, et al. Inflammatory Response and Barrier Dysfunction by Different e-Cigarette Flavoring Chemicals Identified by Gas ChromatographyMass Spectrometry in e-Liquids and e-Vapors on Human Lung Epithelial Cells and Fibroblasts. Appl In Vitro Toxicol 2017;3:28-40.

29 Sussan TE, Gajghate S, Thimmulappa RK, et al. Exposure to electronic cigarettes impairs pulmonary anti-bacterial and anti-viral defenses in a mouse model. PLOS One 2015;10:e0116861

30 Miyashita L, Suri R, Dearing E, et al. E-cigarette vapour enhances pneumococcal adherence to airway epithelial cells. Eur Respir J 2018:51:1701592.

31 McConnell R, Barrington-Trimis JL, Wang K, et al. Electronic Cigarette Use and Respiratory Symptoms in Adolescents. Am I Respir Crit Care Med 2017;195:1043-9.

32 van der Zanden EP, Snoek SA, Heinsbroek SE, et al. Vagus nerve activity augments intestinal macrophage phagocytosis via nicotinic acetylcholine receptor alpha4beta2. Gastroenterology 2009;137:1029-39.

33 Ogunwale MA, Li M, Ramakrishnam Raju MV, et al. Aldehyde Detection in Electronic Cigarette Aerosols. ACS Omega 2017;2:1207-14.

34 Dalleau S, Baradat M, Guéraud F, et al. Cell death and diseases related to oxidative stress: 4-hydroxynonenal (HNE) in the balance. Cell Death Differ 2013;20:1615-30.

35 Poli G, Schaur RJ, Siems WG, et al. 4-hydroxynonenal: a membrane lipid oxidation product of medicinal interest. Med Res Rev 2008;28:569-631.

36 Austin V, Crack PJ, Bozinovski S, et al. COPD and stroke: are systemic inflammation and oxidative stress the missing links? Clin Sci 2016;130:1039-50.

37 Thorley AJ, Tetley TD. Pulmonary epithelium, cigarette smoke, and chronic obstructive pulmonary disease. Int J Chron Obstruct Pulmon Dis 2007;2:409-28.

38 Galvis G, Lips KS, Kummer W. Expression of nicotinic acetylcholine receptors on murine alveolar macrophages. J Mol Neurosci 2006;30:107-8.

39 Kihara T, Shimohama S, Sawada H, et al. alpha 7 nicotinic receptor transduces signals to phosphatidylinositol 3-kinase to block A beta-amyloid-induced neurotoxicity. J Biol Chem 2001;276:13541-6.

40 Kozlowski LT, Mehta NY, Sweeney CT, et al. Filter ventilation and nicotine content of tobacco in cigarettes from Canada, the United Kingdom, and the United States. Tob Control 1998:7:369-75.

41 Benowitz NL, Jacob P. Daily intake of nicotine during cigarette smoking. Clin Pharmacol Ther 1984;35:499-504.

42 Benowitz NL, Hukkanen J, Jacob P. 3rd. Nicotine chemistry, metabolism, kinetics and biomarkers. Handb Exp Pharmacol 2009:192:29-60.

43 Clapp PW, Pawlak EA, Lackey JT, et al. Flavored e-cigarette liquids and cinnamaldehyde impair respiratory innate immune cell function. Am J Physiol Lung Cell Mol Physiol 2017;313:L278-L292. 